\title{
La démarche décisionnelle du gestionnaire en situation complexe : une modélisation
}

\author{
par Marc-André Morency et Jeanne Simard
}

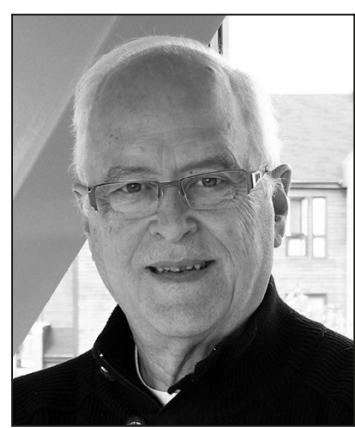

Marc-André MORENCY

M.A., sociologue, professeur retraité

Chicoutimi, Canada

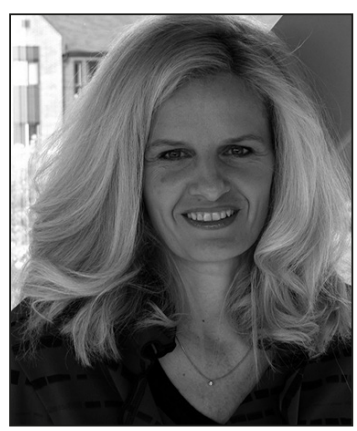

Jeanne SIMARD

LL.D., avocate, F. Adm.A., Professeur-titulaire

Chicoutimi, Canada
"Un problème créé ne peut être résolu en réfléchissant de la même manière qu'il a été créé".

Albert Einstein

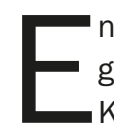
n janvier 2012, le créateur de la pellicule et de la photographie populaire au xIx siècle, la mythique compagnie Kodak, déclare faillite après 131 ans d'existence. Selon une analyse, la firme aurait, de façon délibérée, renoncé à accomplir le virage technologique que la révolution informatique rendait possible. Pourtant Kodak possédait l'appareil photo numérique dès les années 1970, mais n'avait pas cru à son intérêt commercial, le mettant en vente à un prix exorbitant, protégeant du fait même son lucratif commerce de la pellicule. Cette lecture erronée de la réalité de ses clients, alors que la crise et l'inflation grugeaient les revenus de tous les consommateurs, allait créer une opportunité sans pareille pour des concurrents plus innovateurs (Agence-France Presse, 2011). On attribue le même déficit de lecture aux géants américains de l'automobile qui n'ont rien vu venir de la demande d'efficacité environnementale, malgré les avertissements servis par la double crise pétrolière. Pendant que les constructeurs étrangers investissaient dans la production de véhicules plus efficaces, le "Big Three" (GM, Chrysler, Ford) continuait de miser sur les grosses cylindrées énergivores et les modèles lourds, aux antipodes de la demande urbaine mondiale (M. Freyssenet et B. Jetin, 2011).

Ces entreprises eurent en commun qu'elles préféraient conserver les modèles connus plutôt que d'affronter les défis de la complexité, du risque et de l'incertitude engendrés par la présence de nouveaux acteurs dans le marché. Leurs dirigeants ne semblaient alors pas réaliser que la prise de décision face à des situations émergentes comporte toujours un aspect de rupture avec le passé, le dépassement des routines organisationnelles et des stratégies défensives. Les avancées technologiques, les mouvements sociaux, les nouvelles responsabilités légales ou 
déontologiques dans l'esprit du développement durable, obligent désormais à prendre en considération une variété de cadres de référence et de discours pouvant à la limite requérir des solutions radicales, comme le rappelle le célèbre mot d'Einstein. Les solutions novatrices procèdent de variables dont l'analyse repose sur une sensibilité et une maîtrise de connaissances ou de procédures qui ne s'arrêtent pas à la possession préalable de solutions techniques, de séquences d'actions prédéterminées. Plus concrètement, "imaginer les choses autrement " requiert du gestionnaire des capacités d'interprétation et d'anticipation de phénomènes sociaux souvent négligées dans les écoles et facultés de gestion. C'est pourtant sur de telles bases qu'il pourra, en collégialité, mobiliser les solidarités entre les nombreuses parties prenantes d'une organisation, coconstruire les connaissances, et identifier les implications éthiques de la prise de décision. La modélisation que propose ce texte contribue à repérer plus facilement les figures que prend la transformation des manières de penser et d'agir dans la pratique du gestionnaire, tant dans le domaine technoscientifique (discerner ce qui est possible ou impossible), que juridique (ce qui est légal ou illégal), moral ou éthique (ce qui est admissible ou souhaitable). Inspirée par une épistémologie pragmatique et constructiviste, cette modélisation permet de mieux visualiser la forme itérative de la démarche décisionnelle. Cette démarche parcourt quatre moments distincts : 1) l'immédiat, traduisant la conscience habituelle des acteurs sociaux, des professionnels, des gestionnaires; 2) le champ des médiats, c'est-à-dire les outils permettant d'observer, d'analyser, de voir autrement; 3) l'immédiat expliqué, qui résulte de cette activité épistémologique, méthodologique et théorique, substituant une certaine vision des choses à une autre, et enfin ; 4) l'intervention réflexive, qui sur cette base nouvelle, apporte réponse aux défis de la situation, en produisant de nouvelles solutions aux problèmes qui se posaient à la conscience immédiate ${ }^{1}$. La bonne compréhension du caractère en boucle de cette réalité vécue représente une avenue intéressante d'amélioration des formations initiales ou continues des gestionnaires et des professionnels à qui notre société confie des responsabilités aussi importantes. Ces dernières années, la perte de confiance dramatique du public vis-à-vis du milieu des affaires et des gestionnaires en dit long sur les efforts qui devront être déployés pour rectifier une situation de crise qui perdure encore aujourd'hui.

\section{La forme itérative de la prise de décision en situation complexe}

Pour bien comprendre le défi qui se présente au gestionnaire dans une démarche décisionnelle en situation de complexité et de risque, une brève incursion dans l'épistémologie de l'agir professionnel s'impose. Depuis le milieu des années 1970, Donald Schön et Chris Argyris invitent l'ensemble des professionnels (et

1. Jean Piaget avait bien identifié ces trois premiers moments de la démarche scientifique-critique héritée du xixe siècle. non seulement les gestionnaires) à acquérir les habiletés liées à la pratique réflexive. Dans le cadre de cette approche, le praticien déploie des ressources lui permettant d'examiner une situation particulière à la lumière des savoirs scientifiques et techniques disponibles avant de prendre une décision, mais aussi de mettre éventuellement à la disposition des chercheurs les résultats de ses interventions. C'est une formule de co-construction des connaissances analogue à la recherche-action popularisée par le psychosociologue Kurt Lewin. "[...] La théorie et l'action sont toujours étroitement liées : pas d'action sans théorie mobilisée, pas de théorie rectifiée sans action vérifiée; l'insuffisance théorique à résoudre adéquatement une nouvelle situation conduit à de nouvelles pratiques qui, une fois consolidées par l'expérience, devront intégrer le champ théorique et la formation supérieure "(G. Legault et J. Patenaude, 2009, 173).

C'est ainsi que la capacité du gestionnaire d'interpréter, de critiquer, d'anticiper, d'imaginer, de décider et d'agir, s'inscrit de fait au sein d'une séquence décisionnelle circulaire, une forme itérative qui met en jeu les acquis de l'action et ceux du plan théorique. Cette boucle a pour propriété essentielle de déborder la conscience spontanée des situations, en s'inspirant de disciplines et de cadres critiques favorisant de nouveaux aperçus, de nouvelles approches, c'est-à-dire un recadrage de l'action. Une vision réformée ou radicalement nouvelle, convenablement mise en scène, vient nourrir la pratique afin d'améliorer ou de corriger des éléments d'une situation problématique, de réaliser des projets d'affaires. Ce processus, expression de la modernité, n'a pas de fin; aucun processus n'atteint une pure et définitive rationalité.

Cette figure de l'action, forcément simplifiée, met en évidence l'existence et l'interaction de deux domaines d'action : le domaine des pratiques et le domaine de la réflexion théorique. On distingue alors quatre champs, jamais complètement isolés, dont deux appartiennent au domaine de la pratique, impliquant l'ensemble des acteurs sociaux, et deux qui mobilisent avant tout des scientifiques ou des "sages", dans le cadre d'un domaine plus théorique. Cette division en quatre champs typiques ne doit pas conduire à oublier que la vie sociale, une réalité maintenant globale, n'est jamais aussi radicalement séparée ou morcelée. Si le champ théorique consiste à chercher à "comprendre mieux", le champ des pratiques vise "à faire mieux". Comme le rappelle Henry Mintzberg : "[...]une bonne théorie est formidable. Elle explique clairement des choses qu'on n'a pas comprises avant. Je suis un grand amateur de la théorie élégante, mais c'est d'abord l'action qui compte. La théorie suit la pratique et l'influence par la suite" (M. Tremblay, 1999). C'est ce principe même qui est représenté par la figure 1 , synthèse graphique de cette boucle que doivent parcourir les réflexions théoriques et pratiques en saine gouvernance.

La pratique réflexive, si elle s'exerce sur les bases d'une réelle compétence scientifique et pratique, mobilisera bien davantage des compétences en délibération éthique. La prise de décision sera balisée par des normes (légales, déontologiques, économiques, morales) en mouvement, telles qu'elles surgissent dans 
la plupart des sociétés contemporaines, mais surtout, elle saura procéder à une médiation des valeurs en conflit. Ainsi conçue, la pratique réflexive représente de plus en plus l'intégration et l'expression d'une éthique de la responsabilité, d'une pragmatique supérieure.

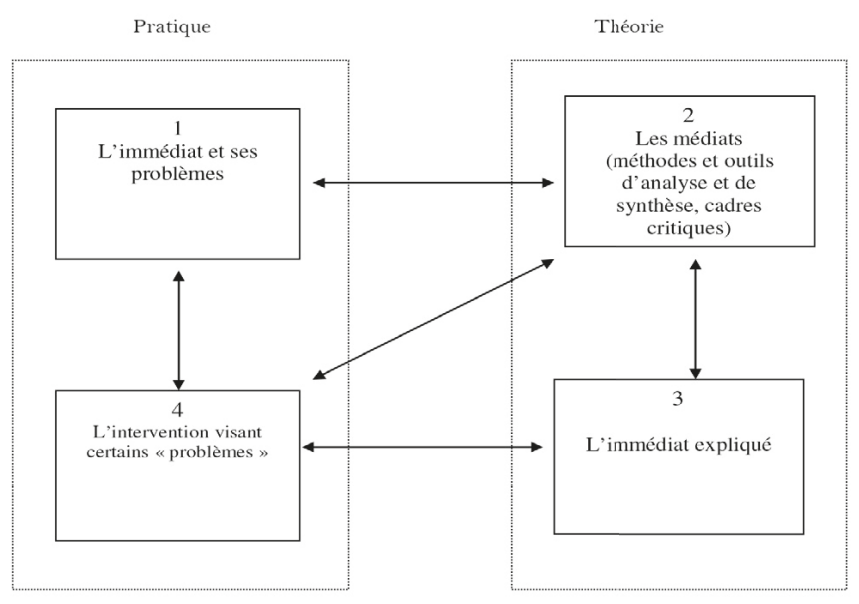

Schéma 1. Le processus décisionnel réflexif et pragmatique

\section{Le champ de l'immédiat}

Le concept d'immédiat représente l'activité habituelle des acteurs sociaux, ce qui inclut l'activité courante des gestionnaires. C'est la réalité telle que nous la percevons spontanément; c'est le monde familier des individus, des groupes, des organisations, des unités techniques de travail, œuvrant dans la quotidienneté. Dans cet immédiat, on trouve donc les innombrables gestes habituels de vie, de travail, d'entraide et de dévouement, sans oublier les aspects moins glorieux du comportement humain. Cette vie commune constitue une "réalité" perçue en tant qu'environnement relativement stable. De nombreux savoirfaire et pratiques des gestionnaires peuvent être accomplis en mode de "pilotage automatique", sans qu'il y ait de remise en question ou innovation. Des tâches telles que préparer certains documents, dresser des budgets, négocier avec les fournisseurs, sont assimilables à des processus courants. Engagés dans la routine de leur vie, les acteurs ont rarement une vision globale et claire de toutes les raisons qui les font agir. De fait, la plupart de ces actes ordinaires ou récurrents se déroulent dans un état préréflexif de semi-conscience, permettant à l'esprit d'économiser ses ressources cognitives en s'appuyant simplement sur des formules et règles générales issues de l'habitude, de la culture ou de l'analogie. Diane Girard, s'appuyant sur les études du psychologue américain Gary E. Klein et celles de la professeure en management Dolly Chugh, illustre bien ce phénomène : "Most decisions made every day by people in organizations can be characterized as "routine," and more or less spontaneous. In fact, as G. Klein (1989) concluded from interviews with 150 people making decisions, "relatively few decisions are made using analytical processes such as generating a variety of options and contrasting their strengths and weaknesses" [...]. The busier people are, the more likely they are to rely on a faster, effortless, and routine decision-making process. With the frantic pace of today's workplace, it is thus to be expected that people often rely on such modes of thinking (Chugh 2004)" (D. Girard, 2012, 120). C'est précisément eu égard à l'habitude que la vigilance doit s'imposer, car cet immédiat est traversé par une multitude de représentations mensongères ou trompeuses. Les idéologies politiques, économiques, religieuses, travaillent les représentations des individus pratiquement à leur insu; elles ont pour propriété d'interpréter le monde, mais avant tout d'en déguiser la nature sociale et ses rapports avec la nature. Les idéologies imposent à la conscience "une lentille à travers laquelle voir le monde, un ensemble de croyances auxquelles on adhère si fort qu'il n'est presque pas besoin de confirmation empirique" (J. Stiglitz, 2003). Elles s'avèrent donc très utiles et intellectuellement confortables lorsque vient le temps de rationaliser, de justifier, a posteriori, les fausses solutions données aux problèmes. Comme le mentionne le philosophe Daniel Innerarity : "Lorsqu'on ne dit que ce qu'on attend de nous, ce qui est approprié et en accord avec l'opinion dominante, on ne contribue en rien à la compréhension de la réalité sociale. Et c'est cette inauthenticité même qui éveille l'intuition que la vérité doit être recherchée justement en dehors de l'unanimité, du lynchage et de l'adulation qui gouvernent l'opinion publique, dans un ailleurs non contrôlé par les arguments de ce qui est opportun ou des réactions concertées du politiquement correct; un ailleurs où ce qu'on dit aura véritablement été pensé" (D. Innerarity, 2012, 10).

Ainsi, les gestionnaires peinent souvent à s'extirper du fait que le libéralisme et sa récente reformulation, le néolibéralisme, les mains invisibles du marché et la globalisation se présentent comme des phénomènes incontournables, comme des réalités qui s'imposent sans alternative possible. De façon plus simpliste, diverses idéologies héritées du xIx ${ }^{\mathrm{e}}$ siècle persistent à faire considérer des groupes, d'autres ethnies, comme moins intelligents, plus paresseux et causes premières de leur mal développement. On s'illusionne à croire, comme le rappelle Étienne Perrot, que le progrès porte "la promesse de pouvoir réparer dans l'avenir les dommages causés par l'ignorance d'aujourd'hui " (E. Perrot, 2007, 19).

Ces fausses croyances, ces idéologies, ces raisonnements défectueux, peuvent avoir des effets très insidieux dans la vie quotidienne des gestionnaires, se manifestant par une faiblesse de l'esprit critique, de l'agir créatif et de la prise de décision éthique. Cette passivité engendre alors une spirale de sous-développement, laquelle concrètement peut se traduire par le fait que les gestionnaires privilégient le profit à court terme, délaissant ainsi bien souvent la pleine mobilisation des travailleurs, la relation contractuelle durable avec les clients, et l'intérêt de la société en général.

Pour toutes ces raisons, au-delà de leurs intuitions, de leurs dons personnels, les gestionnaires ont à passer au crible, à redéfinir le "champ de l'immédiat ", les problèmes qu'il comporte, en bref, 
ils ont à s'approprier les outils de contestation du sens commun. Ils doivent en quelque sorte franchir la barrière de l'immédiat, entrer dans des espaces moins confortables, pratiquer l'art du doute méthodique. II est significatif que l'on trouve de plus en plus dans les lois québécoises et canadiennes, une obligation à franchir cette barrière. À titre d'exemple, l'application en matière environnementale du principe de précaution "suppose une infinie volonté de savoir" afin de "prendre les devants, d'aller au-devant du risque, de la menace avant qu'elle ne se réalise " (F. Ewald, C. Gollier, N. Sadeleer, 2008, 30). C'est la mise en œuvre de ces "médiats" ou moyens critiques, qu'il faut maintenant aborder.

\section{Le champ des médiats : les outils et méthodes en science}

La prise de distance, c'est le défi principal que comporte nécessairement l'activité scientifique (sciences pures, sciences humaines et sociales, épistémologie). Au cours des âges, les chercheurs de vérité ont patiemment réussi à structurer un regard critique, à élaborer des théories de la connaissance, des méthodes et techniques d'observation ou de mesure. Jean Piaget appelait médiats les outils permettant d'observer, de voir autrement, et surtout d'expliquer, révélant de nouveaux rapports entre les phénomènes. Ces nouvelles connaissances permettent éventuellement de faire autrement. Par ces moyens épistémologiques (façons dont nous venons à la connaissance; positivisme, constructivisme, behaviorisme, etc.), méthodologiques (observation, recueil des données, enquêtes, études de cas, etc.), et conceptuels (nos tentatives d'explication), les anciennes visions et théories sont soumises à l'épreuve de la vérification. On fait opérer ce que Karl Popper appelait le critère de falsification; si une affirmation ne peut être confirmée, ni ne peut être infirmée, elle ne saurait constituer un objet ou un résultat avéré du discours scientifique. Toute proposition de nature scientifique doit ainsi se soumettre à la critique de faits construits et de la logique. Inévitablement, elle se trouvera remplacée par une conception de meilleure qualité, souvent capable d'embrasser une réalité plus complexe. On est en présence d'un cycle, d'une spirale permanente qui produit l'émergence de ces nouvelles connaissances, lesquelles prédominent jusqu'à ce que techniques et paradigmes montrent leurs limites et nécessitent d'être à leur tour remplacés. C'est la nature du scientifique de se libérer des visions imposées par les pouvoirs, les mentalités, de se libérer de ses propres lacunes. Lorsque cette libération ne réussit pas, on se retrouve dans le champ socialement contrôlé des techniques... dans l'immédiat.

\section{L'immédiat expliqué}

La notion d'" immédiat expliqué " représente le produit de l'activité épistémologique, méthodologique et théorique, qui réussit à renverser une certaine vision des choses, à en produire une autre et à la diffuser de façon à la faire accepter. Les décou- vertes scientifiques présentent un intérêt si elles fournissent des savoirs, des explications, des esquisses de solutions. Elles redéfinissent nos façons d'interpréter le monde, mais aussi notre capacité d'adaptation et de transformation de la "réalité". "Une bonne théorie est formidable", rappelle Henry Mintzberg : "Elle explique clairement des choses qu'on n'a pas comprises avant " (M. Tremblay, 1999). Elle permet de nous débarrasser de nos œillères, d'envisager de nouvelles approches et de trouver éventuellement solution à quelque problème. Cette production rend possible l'élargissement, selon Amartya Sen, (2010), de la sphère de nos "capabilités".

Pour donner exemple de ce processus créatif, il est toujours plus facile de rappeler les productions plus anciennes des sciences pures. Elles sont plus spectaculaires, car elles sont maintenant mieux connues dans leurs effets. Les plus récentes font parfois l'objet d'une grande publicité, comme en témoigne la " mise en évidence" du Boson de Higgs, pourtant responsable de l'aspect gravitationnel de la réalité. Ainsi, l'utilisation de nouveaux concepts et outils de mesure, comme les télescopes et le calcul, a permis aux créateurs de l'astronomie moderne de démontrer que la terre est sphéroïde, qu'elle est, comme les autres planètes, en mouvement orbital autour du soleil. L'ancienne vision du cosmos (la Terre étant vue comme le centre immobile de couches sphériques d'univers) en a été bouleversée, obligeant à reconfigurer d'anciennes représentations religieuses sur la place de l'homme dans l'univers.

Les recherches scientifiques dans les disciplines telles que les sciences économiques et sociales (gestion, psychologie, sociologie, linguistique, communication, droit, etc.) reçoivent peut-être moins de publicité, mais elles n'en sont pas moins importantes. Ainsi la recherche en sciences humaines a permis de mieux comprendre les processus décisionnels, les jeux d'influence ou de coopération entre les acteurs, l'importance des représentations dans la création de la réalité, les problématiques de développement et de mal développement. Plus spécifiquement, en sciences économiques et administratives, divers travaux ont amené les gestionnaires à mieux appréhender leur rôle, le traitement de la complexité et de la responsabilité, les nouveaux aspects de la gouvernance des organisations et des projets. Les théories de l'action, en sociologie, ont permis d'élucider la variété des déterminants et caractéristiques des comportements des acteurs au sein de leur organisation ${ }^{2}$. La théorie des contraintes, appliquée à la gestion de projet, a fait surgir "l'approche chaîne critique". La théorie de la structuration apporte des réponses descriptives, et même normatives, favorisant l'évolution des entreprises, une meilleure lecture des mécanismes organisationnels et humains à l'origine des routines

2. Cabé Marie-Hélène, Robert-Tanguy Philippe. L'intervention sociologique : (re)donner du sens à des pratiques gestionnaires. Sociologies pratiques, 2008, vol. $1 n^{\circ} 16$, p. 39-54; Delahaye Hélène. Sociologue en entreprise : réflexions sur la complémentarité des savoirs. Sociologies pratiques, 2008, vol.1, $n^{\circ} 16$, p. 55-62; Huhalde Marc. Quand gestion rime avec confusion. Sciences humaines, 2007, vol. 2, n 179. 
et de leur évolution, des conditions et modalités de changement des structures elles-mêmes ${ }^{3}$.

Dans ce champ des médiats expliqués la science présente ce qui est pensable ou possible de faire aujourd'hui. Si le champ des "compossibles" peut ainsi s'élargir, certaines découvertes scientifiques demeurent parfois "sur les tablettes" durant des années, sinon des décennies, sans qu'on puisse leur trouver à court terme une quelconque utilité. L'exemple le plus célèbre est sans aucun doute la découverte des mathématiques binaires, inutiles pour nos calculs habituels, mais qui sont à la base du fonctionnement de nos ordinateurs. Les découvertes fondamentales représentent très souvent des changements pratiques à survenir plus tard, dont l'importance sera énorme. La situation présente fait espérer que la physique et la chimie permettront éventuellement de résoudre le déficit énergétique qui afflige présentement nos sociétés tributaires du pétrole, ou de son absence.

Cette vision optimiste du recours à la science pour transformer positivement la société, régler les problèmes de l'immédiat, ne doit pas occulter la présence de nombreuses dérives éthiques qui peuvent réduire et assimiler les activités scientifiques à une pluralité de techniques et d'utilités plus ou moins serviles à l'égard du politique, du militaire, des besoins ressentis dans l'entreprise. À la limite, lorsque l'activité scientifique est asservie aux intérêts économiques ou militaires des dirigeants, du matérialisme ambiant, elle se rend indistincte de la pratique, retombant au rang de simple outil idéologique, un aspect de l'immédiat voire de la tragédie. C'est ce que le philosophe André Comte-Sponville appelle la Loi de Gabor qui, par exemple, a vu le nitrate, un formidable engrais à l'origine de la révolution verte, se transformer en explosif ou en gaz mortel (le fameux gaz moutarde de la Grande Guerre). "Tout le possible sera fait, toujours. [...] Or, il se trouve que le possible aujourd'hui devient particulièrement effrayant [...]. Le progrès technologique n'est pas une garantie. II peut se retourner contre nous, au point de mettre en cause l'existence même de l'humanité..." (A. Comte-Sponville, 2004, 50).

En tout état de cause, l'ensemble des productions scientifiques constitue un bagage non seulement de savoirs disponibles, mais aussi une base d'intervention pour l'innovation, la solution de certains problèmes immédiats. Elles entrent dans la formulation d'interventions, dans une nouvelle réflexivité. Alors, les balises juridiques, morales et la réflexion éthique, qui ont toujours leur place dans l'activité scientifique, paraissent encore plus indispensables en raison des impacts de toute intervention modifiant la vie sociale, les rapports de pouvoir.

\footnotetext{
3. Giddens Anthony. Structuration theory: past, present, future. In Bryant Christopher G. A., Jary David et al. Giddens' theory of structuration : A critical appreciation, Bryant Christopher G. A., Jary David (dir.), London, New York : Routledge, 1991, chapitre 8, pp. 201-221.
}

\section{L'intervention réflexive}

Dans une situation marquée par le besoin de nouvelles approches ou solutions, le gestionnaire apparaît comme un intervenant possible, et non plus comme simple exécutant. Dans un plus large contexte, il doit pouvoir acquérir, puis faciliter la transmission des découvertes au moyen d'interventions destinées à résoudre quelques problèmes concrets. Le gestionnaire peut viser à améliorer la réalité des gens par une nouvelle approche de gouvernance, avec de nouveaux processus, de nouveaux produits. II peut contester certaines conceptions ou idéologies qui freinent l'innovation et la délibération éthique. En bref, le rôle de l'intervenant est de changer les choses, de penser autrement, de critiquer les obstacles, d'aider au développement et au changement de culture d'une organisation privée ou publique, d'un milieu, mais aussi de la société. L'objectif est ultimement de résoudre les problématiques qui surgissent dans l'"immédiat", tel que nous l'entendons ici. Le défi que représentent l'actuelle crise énergétique et ses impacts sur le climat planétaire offre un exemple des ruptures à réaliser. "In early 2007 the UN Intergovernmental Panel on Climate Change, including the world's leading scientists on the subject, issued a report documenting that climate change exists, that human activity is a key driver, and that the many resulting likely threats to humans include water shortages, dropping crop yields, and rising sea levels. The report resulted from a review of hundreds of scientific studies and complex supercomputer simulations. Many American companies, including General Electric, Wal-Mart, and DuPont Chemical, are making sustainability an essential part of their business strategy. This is largely because executives of these companies agree with the scientific consensus " (L. Treviño, G.R. Weaver G. R., 2003, 334).

Comme "accompagnateur", "facilitateur ", "coach ", "animateur", peu importe comment on le désigne, le gestionnaire a, de fait, la responsabilité d'intégrer dans la pratique sociale les nouvelles interprétations issues du travail scientifique. II participe au travail de co-création collective. Par exemple, il se doit de repérer les connaissances disponibles au sein du personnel de son organisation, de le mobiliser, de l'écouter; il comprend l'importance de la formation continue pour les professionnels intégrés dans les divers services. Le défi est de faire en sorte que les savoirs intègrent la pratique et que la pratique puisse favoriser la production des savoirs. Dans ce processus, le gestionnaire doit intervenir de façon proactive, et non s'avérer un obstacle à la pleine mobilisation des connaissances et des capacités du personnel à son service. A contrario, dans la foulée de la logique néolibérale, la mode de réingénierie par compression du personnel fut une faute commise au nom de la maximisation de la valeur des actionnaires. La crise de 2007-2008 a révélé toutes les dérives portées par les énormes stimuli offerts à la haute direction, sous forme de "stock options". Henry Mintzberg l'a constaté : "C'est affreux! Ils ont détruit beaucoup de capacités dans les entreprises. La connaissance des entreprises est dans les mains des cadres intermédiaires et on les a virés" (M. Tremblay, 1999). 
Le gestionnaire doit aussi comprendre les phénomènes de blocage, de résistance au changement. Dans le domaine des sciences de l'homme et de la société, les théories abondent, mais présentent souvent de plus grandes difficultés d'application en raison de la rareté d'expériences contrôlées, de moyens plus limités de persuader, de la résistance de l'immédiat. Lorsqu'on coupe le métal au laser, ce métal n'offre pas de contestation. En sciences humaines, en sciences de la gestion, c'est tout autre chose que de proposer des innovations venant remplacer des habitudes bien ancrées, défendues par des intérêts vigilants. Plusieurs études démontrent que les plus gros blocages trouvent leur origine dans la culture organisationnelle qui s'impose subrepticement à la conscience des agents sociaux $x^{4}$.

Le gestionnaire doit apporter solution à une importante difficulté : la gestion des balises devant présider à l'usage des connaissances scientifiques dans la pratique. Le praticien (gestionnaire) réflexif, et le milieu dans lequel il agit, ne peuvent jamais être considérés comme de simples réceptacles passifs des lumières de la science. Les fines observations de $D$. Schön et $\mathrm{Ch}$. Argyris, et d'autres théoriciens-praticiens de la scienceaction ont permis de réaliser que de l'immédiat surgissent des problèmes, des questions, des valeurs qui doivent orienter la production "scientifiquement-éclairée" de solutions nouvelles. L'État a bien établi certaines balises en adoptant des lois et des règlements (on pense au développement des notions de prudence, de prévention, de précaution) mais ce n'est pas suffisant. En effet, les interventions, comme les travaux scientifiques en général, ne peuvent être dissociés de la morale (l'admissible et le souhaitable, que l'on retrouve bien souvent concrétisés dans la déontologie professionnelle) et surtout d'une éthique du mieux-être collectif.

Dans l'intervention, l'intégration des perspectives et des normes, qu'elles soient techniques, scientifiques, ou plus proprement juridiques, morales ou éthiques, ne va jamais sans exiger un travail décisionnel impliquant un haut niveau de conscience. Le choix des solutions engage la personnalité des partenaires. On comprendra qu'une organisation, une entreprise, n'est pas un agent éthique, ni l'agent d'une soi-disant responsabilité sociale. II n'y a de morale ou d'éthique que pour les individus, les dirigeants d'une organisation. En raison des conflits opposant les normes, les perspectives, les résistances propres aux divers intérêts, on peut estimer à bon droit que la "sur-ordination" aux valeurs, la hiérarchisation des valeurs, impliquent un engagement émotionnel, esthétique, intuitif, développemental, allant bien au-delà des exigences scientifiques et techniques, de savoirs toujours perfectibles, au-delà des attentes normatives usuelles. Pour le gestionnaire, il s'agit d'avoir pleinement conscience des éléments pertinents à la prise de décision, des aspects complexes de la prise de responsabilité. Ce qui pose la question de la formation des intervenants, des gestionnaires

4. Schein Edgar Henry. Organizational culture and leadership. 3rd ed. San Francisco : Jossey-Bass, 2006, 464 p.; Girard Diane. Culture organisationnelle, contexte d'affaires et prise de décision éthique. Gestion, 2007, vol. 32, no 1, p. 101-111. en particulier, en vue de la maîtrise de la boucle décisionnelle que nous venons de déterminer.

\section{Conclusion}

Comme il n'y a que 24 heures dans une journée, lorsque vient le temps d'agir, un gestionnaire seul ne peut, même avec la meilleure volonté du monde, avoir en vue la réalité globale avec "les interactions complexes qui la caractérisent" (B.K. Murphy, 2001, 173). Pour cette raison, il importe d'avoir à disposition ou de développer des outils, des mécanismes, des stratégies permettant de relier les actions, les connaissances des parties prenantes d'une organisation. Pour plusieurs (c'est aussi notre position), une telle évolution passe par l'instauration d'une gouvernance collaborative et réflexive dans les organisations. Est dépassé le modèle voulant qu'un "Grand leader omniscient" soit à la barre du vaisseau. Plusieurs auteurs privilégient actuellement un modèle de "stewardship" pour caractériser la coordination efficace de cet apprentissage collectif au sein d'une organisation. Le "stewardship" représente une sorte de pilote en modification continue à mesure que le groupe apprend de ses erreurs et modifie ses arrangements et les fardeaux de la charge en conséquence" (G. Paquet, 2012). Toujours selon Gilles Paquet : "Ce maintien de la capacité à apprendre, à se réoutiller, à se restructurer, de même qu'à recadrer ses perspectives, est le défi crucial de cette gouvernance collaborative et réflexive : intégration de la connaissance et apprentissage dans l'action; capacité à prospecter les effets de systèmes à long terme; adaptation des stratégies et des structures; définition participative et itérative des grandes directions; et développement interactif des stratégies" (G. Paquet, 2011, 57).

Dans cette perspective, il s'agit de réinventer de nouvelles façons d'apprendre en entreprise et de faciliter l'émergence d'une intelligence collective. Plusieurs approches utilisables sont bien connues ou largement étudiées; pensons aux formules de symposiums mixtes, de recherche-action, de collaboration université-milieu, aux méthodologies orientées à la résolution de dilemmes éthiques ou déontologiques, etc. À tout le moins, notre schéma du processus décisionnel réflexif et pragmatique devrait permettre aux gestionnaires de mieux cerner le circuit sans fin que les processus complexes empruntent désormais, c'est-à-dire ces cycles décisionnels plus ou moins coordonnés formant la trame de la vie contemporaine. Un tel schéma ne se présente pas comme une proposition facultative, un utilitaire quelconque pour la prise de décision : il représente selon nous une dimension incontournable de la réalité en cours. Il est, pour autant que nous puissions en juger, une image de la réalité telle que l'œil sociologique peut aujourd'hui la représenter. Cette réflexion nous semble pertinente, aussi bien pour la communauté globale elle-même que pour les responsables du "stewardship" de la gouvernance. Écarter les citoyens de ces connaissances représente un facteur de sous-développement, de non-mobilisation optimale des "ressources". L'autonomie 
réflexive du gestionnaire requiert l'assimilation de savoirs produits par diverses disciplines ou professions, ce qui lui permet de développer sa conscience des idéologies, des idées fausses et favorise de nouveaux concepts de réalité. En développant sa compétence face à la complexité, le gestionnaire saura mieux participer à l'animation des changements que vont requérir les nombreux processus globalisés de la planète.

\section{Bibliographie}

Agence France-Presse, Presse Canadienne. "Faillite appréhendée: Kodak s'effondre à la Bourse de New York". Le Devoir, samedi 1er octobre 2011, p. C3.

Bourdieu Pierre, Passeron Jean-Claude, Chamboredon Jean-Claude. Le métier de sociologue. Paris : Mouton, 1973, 357 pages.

Cabé Marie-Hélène, Robert-Tanguy Philippe. "L'intervention sociologique : (re)donner du sens à des pratiques gestionnaires". Sociologies pratiques, 2008, vol. 1 n 16, p. 39-54.

Carre Philippe, Charbonnier Olivier, Les apprentissages professionnels informels. Paris : L'Harmattan, 2003, 305 pages.

Chugh Dolly. "Societal and managerial implications of implicit social cognition : Why milliseconds matter". Social Justice Research, 2004, vol. $17, n^{\circ} 2$, p. 203-222.

Comte-Sponville André. Le capitalisme est-il moral? : sur quelques ridicules et tyrannies de notre temps. Paris : Albin Michel, 2004, 236 pages.

Cunha Flavio, Heckman James J. "The Economics and Psychology of Inequality and Human Development". Journal of the European Economics Association, 2009, vol. 7, p. 320-364.

Déchaux Jean-Hugues. "L'action rationnelle en débat. Sur quelques contributions et réflexions récentes". Revue française de sociologie, 2002, vol. 43, n³, p. 557-581.

Delahaye Hélène. "Sociologue en entreprise : réflexions sur la complémentarité des savoirs". Sociologies pratiques, 2008, vol.1, n 16 , p. 55-62.

Desaulniers Marie-Paule, Legault Georges A. Crise d'identité professionnelle et professionnalisme. Sainte-Foy (Québec) : Presses de l'Université du Québec, 2003, 226 pages.

Desrosiers Éric. "Temps durs pour les théories. La crise a durement entaché la renommée des sciences économiques". Le Devoir, 21 juillet 2009, p. B1.

Ewald François, Gollier Christian, Sadeleer Nicolas (de). Le principe de précaution. Paris : Presses Universitaires de France, 2008, 127 pages. Freyssenet Michel, Jetin Bruno. "Conséquence de la crise financière ou crise d'une forme de capitalisme : la faillite des Big Three". Revue de la régulation [En ligne], 2011, vol. 9, no 1, [consulté le 14/09/2013]. Disponible à l'adresse : http://regulation.revues.org/9233

Giddens Anthony. "Structuration theory : past, present, future". In : Bryant Christopher G. A., Jary David et al. Giddens' theory of structuration : A critical appreciation, Bryant Christopher G. A., Jary David (dir.), London, New York : Routledge, 1991, chapitre 8, pp. 201-221.

Girard Diane. "Making Ethical Decisions". In : Kissick, W. Peter et al. Business ethics : concepts, cases, and Canadian perspectives, Kissick W. Peter (dir.). Toronto : Emond Montgomery Publications, 2012, chapitre 9, p. 119-137.

Girard Diane. "Culture organisationnelle, contexte d'affaires et prise de décision éthique". Gestion, 2007, vol. 32, n 1, p. 101-111.

Innerarity Daniel. La société invisible. Québec : Presses de l'Université Laval, 2012, 163 pages.

Klein Gary A. "Recognition-primed decisions". In : Klein, Gary A. Advances in man-machine systems Research, Ed. W.B. Rouse, Greenwich, CT : JAI Press, 1989, P. 47-92.
Legault Georges A., Patenaude Johanne. "Le déplacement de la normativité en éthique de la recherche. Enjeux de gouvernance". In : Marc Maesschalck (éd.), Éthique et gouvernance. Les enjeux actuels d'une philosophie des normes. Hildesheim : Georg Olms Verlag, 2009, p. 171-206.

Le Moigne Jean-Louis, Morin Edgar. Intelligence de la complexité : épistémologie et pragmatique. La tour d'Aigues: Éditions de l'aube, 2007, 457 pages.

Levy Joseph Josy, Bergeron Michel. "Éthique de la recherche et responsabilité sociale du chercheur : Perpectives canadiennes et québécoises ". Éthique publique, 2010, vol. 12, n 1, p. 287-297.

Lewin Kurt. Field theory in social science : selected theoretical papers. Westport, Ct : Greenwood Press, 1975, 346 pages.

MacDonald Chris. Critical "Thinking for Business Ethics.", In Kissick, W. Peter et al. Business ethics : concepts, cases, and Canadian perspectives, Kissick W Peter (dir.). Toronto : Emond Montgomery Publications, 2012, chapitre 3, p. 27-40.

Mintzberg Henry. Structure et dynamique des organisations. Paris : Éditions d'organisation, Montréal : Agence d'Arc, 1982, 434 pages.

Mintzberg Henry, Ahlstrand Bruce, Lampel Joseph. Safari en pays stratégie : l'exploration des grands courants de la pensée stratégique. Paris : Village Mondial, 1999, 423 pages.

Morin Edgar. Introduction à la pensée complexe. Paris : Édition du Seuil, 2005, 271 pages.

Murphy Brian K. De la pensée à l'action. La personne au coeur du changement social. Montréal : Écosociété, 2001, 208 pages.

Osty Florence, Sainsaulieu Renaud, Uhalde Marc. Les mondes sociaux de l'entreprise, Paris : La Découverte, 2007, 398 pages.

Paquet Gilles. La gouvernance collaborative : un antimanuel. Montréal : Liber, 2011, 291 pages.

Paquet Gilles. "Gouvernance et éthique ". In : Paquet Gilles. Gouvernance Gilles Paquet governance [en ligne]. [consulté le 14/09/2012]. Disponible à l'adresse : http://www.gouvernance.ca/publications/12-01.pdf

Perrot Etienne. L'art de décider en situations complexes. Paris : Desclée de Brouwer, 2007, 290 pages.

Piaget Jean. Épistémologie des sciences de l'homme. Paris : Gallimard, 1970, 380 pages.

Popper Karl Raimund. La connaissance objective. Paris : Aubier, 1991, 578 pages.

Reynolds Scott J. "A neurocognitive model of the ethical decisionmaking process : Implications for study and practice". Journal of Applied Psychology, 2006, vol. 91, n 4, p. 737-748.

Sainsaulieu Renaud. "Préface". In : L'intervention sociologique en entreprise. De la crise à la régulation sociale, Uhalde Marc (dir.), Paris : Desclée de Brouwer, 2001, p. 11.

Schein Edgar Henry. Organizational culture and leadership. 3rd ed. San Francisco : Jossey-Bass, 2006, 464 pages.

Schön Donald, Argyris Chris. Theory in Practice : Increasing Professional Effectiveness. San Francisco, California : Jossey-Bass Publishers, 1974, 224 pages.

Sen Amartya. L'idée de justice. Paris : Flammarion, 2010, 558 p.

Stiglitz Joseph. Quand le capitalisme perd la tête. Paris : Fayard, 2003, 416 pages.

Tremblay Miville. "Le safari d'Henry Mintzberg en pays stratégique”. La Presse, vendredi 23 avril 1999, p. C1.

Treviño Linda K., Weaver Gary Richard. Managing ethics in business organizations : a social scientific perspectives on business ethics. Stanford, California : Stanford Business Books, 2003, 364 pages.

Uhalde Marc. "Quand gestion rime avec confusion". Sciences humaines, 2007, vol. 2, n 179 . 